\title{
Integration of geotechnical design and analysis processes using a parametric and 3D-model based approach
}

\author{
M. Obergrießer ${ }^{1}$, T. Euringer ${ }^{2}$, A. Borrmann ${ }^{3}$ and E. Rank ${ }^{4}$ \\ ${ }^{1}$ Research Assistant, Construction Informatics Group, Regensburg University of \\ Applied Sciences, Germany. 93049 Regensburg, Prüfeninger Str. 58, Tel. +49 941 \\ 943 1222, Fax +49941943 1426, Email: mathias.obergriesser@hs-regensburg.de \\ ${ }^{2}$ Professor of Construction Informatics, Regensburg University of Applied Sciences, \\ Germany. 93049 Regensburg, Prüfeninger Str. 58, Tel. +49 941943 1226, Fax +49 \\ 941943 1426, Email: thomas.euringer@hs-regensburg.de \\ ${ }^{3}$ Professor, Computational Modeling and Simulation Group, Technische Universität \\ München, Germany. 80290 Munich, Arcisstr. 21, Tel. +49 89289 25117, Fax +49 89 \\ 289 25051, Email: andre.borrmann@tum.de \\ ${ }^{4}$ Professor, Chair for Computation in Engineering, Technische Universität München, \\ Germany. 80290 Munich, Arcisstr. 21, Tel. +49 89289 23048, Fax +49 89289 \\ 25051, Email: ernst.rank@tum.de
}

\begin{abstract}
This paper investigates ways to improve the integration between the geotechnical and infrastructural designing, modeling and analyzing processes. Up till now these three planning stages have been executed separate and without data exchange between each other. This separation leads to a time-consuming and expensive manual re-input of geometric and semantic data. Currently, roads are designed using a traditional approach which is based on various $2 \mathrm{D}$ drawings and is focused on the roadway itself. Additional geotechnical conditions such as the slope angle of the dam or the position of a retaining wall are not considered. To solve these problems, a new parametric and 3D-model based approach has been developed in the research project 'ForBAU - The virtual construction site'. This new approach is based on the traditional 2D infrastructure planning process which is extended by a new parameterized 3D modeling concept. Different open data formats such as LandXML and GroundXML allow data integration with both, a parametric Computer Aided Design (CAD) system and geomechanical engineering software. An automatic update function ensures data flow without loss of information. Use of this new approach will accelerate the infrastructure design and provide a parametric 3D-model approach which will close the gap between the geotechnical and infrastructure planning process. This paper strives to provide detailed information about this new integration concept and gives an overview on the various implementation steps.
\end{abstract}




\section{Motivation}

How can we improve or optimize the construction planning process? Most engineers have to deal with this question in the hope to make their work more economical and efficient. In some Architecture Engineering Construction (AEC) sectors such as structural engineering, several optimization approaches exist. One such approach is Building Information Modeling (BIM) (Eastman et al., 2008). BIM is based on a consistent and central 3D data model that provides engineers with the required building information data during the whole lifecycle of the construction. Furthermore, it improves the collaboration between all of the involved planning companies and processes. Up till now such an approach has not existed in the civil engineering sector and especially not in the infrastructural and geotechnical planning processes. Each sector devises its planning tasks alone and all without any information transfer to other planning fields (Kaminski, 2010). For example, the designing of a roadway requires a lot of information about the positions of existing buildings, the surface of the environment or the conditions of the subsoil. Most of this data can be provided in a digital format. The Infrastructure Engineer however, only re-uses a very small part of this data. Another problem exists in the generation of the physical model for the geotechnical analysis. In the current planning process, the engineer has to integrate the required geometric and semantic information into the system manually. Before he can generate the analysis model, the engineer has to interpret the geometrical information of the infrastructure cross-section plan. The appending semantic information is contained in the ground expertise (Figure 1). Afterward he uses this interpreted information to create a $2 \mathrm{D}$ cross-section which is significant to the geotechnical structural analysis.

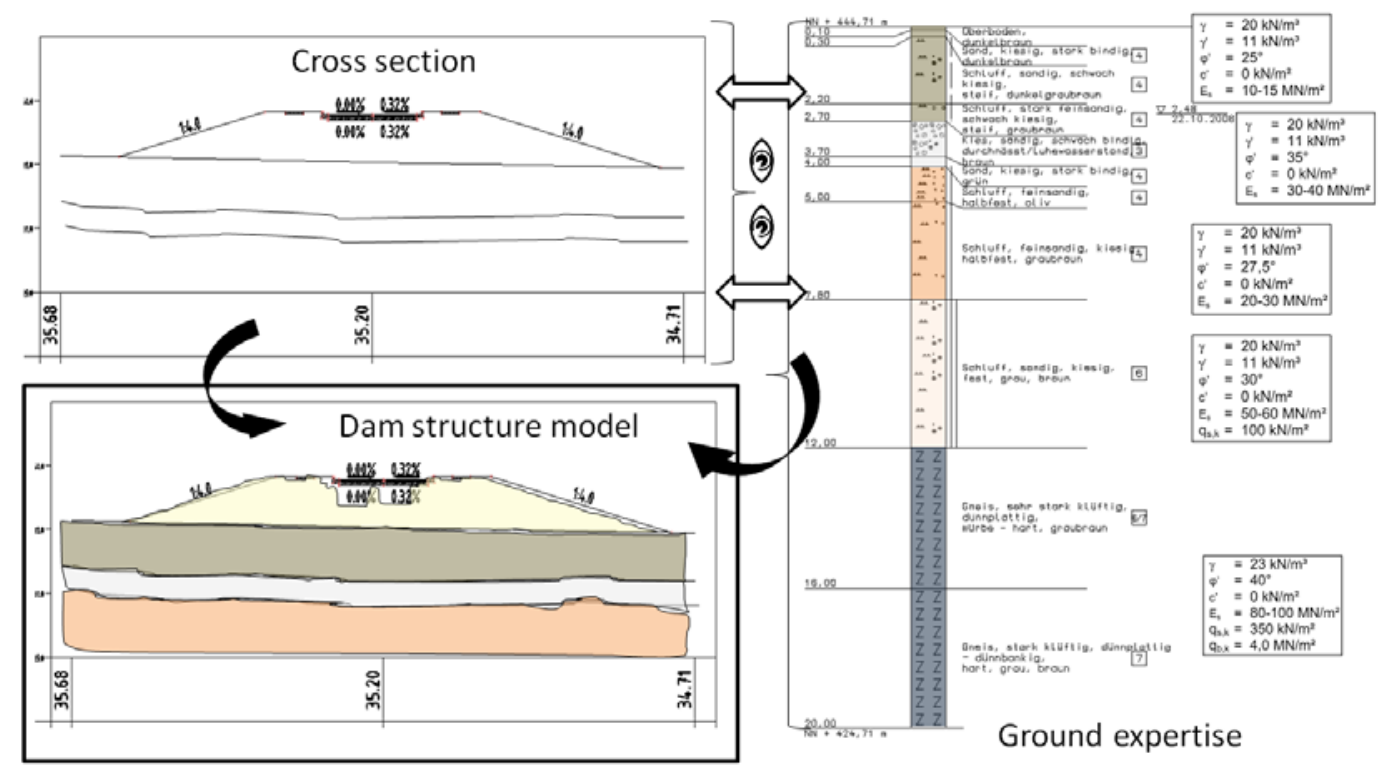

Figure 1. Required datasets for the geotechnical analysis model

The aforementioned issues lead to an inefficient and cost intensive planning process. This paper presents a novel concept for improving and automating the infrastructural and geotechnical planning processes by developing an approach that ensures a conti- 
nuous geometrical and semantic data exchange, realizes a parameterized 3D infrastructure model and connects the different planning processes by using a parametric concept.

\section{Parametric 3D-model based approach}

The following paragraphs describe the implementation of a concept that allows an integrated geotechnical and infrastructural design and analysis process based on a parametric 3D-model. The paper will present a short overview on the available parametric $3 \mathrm{D}$ modeling systems and data exchange formats. It also will explain the new concept in detail and will finally discuss some topological problems that arose during the development process.

\section{Parametric 3D modeling systems}

Parametric modeling means that an object is not created with a fixed geometry however its dimensions are described by free parameters. Furthermore the different values of single parameters can be linked by arithmetical expressions. In the example displayed in Figure 2, the parameter 'area' is given by the multiplication of the parameter 'length' and 'width'. The use of a parametric approach allows updates to the whole geometry by changing one parameter or constraint. It is possible to distinguish two versions of parametric modeling (Eastman et al., 2008). The first one only describes constraints within one object. In this case the objects are instances of predefined element classes. The second kind of parametric modeling is highly important for the modeling of geotechnical and infrastructural buildings like roadways, railways or bridges. It illustrates the position and form of individual geometric objects with reference to other objects. Changing the value of a parameter either results in the system generating a warning, if rules are violated, or in an automatic update of the entire model.
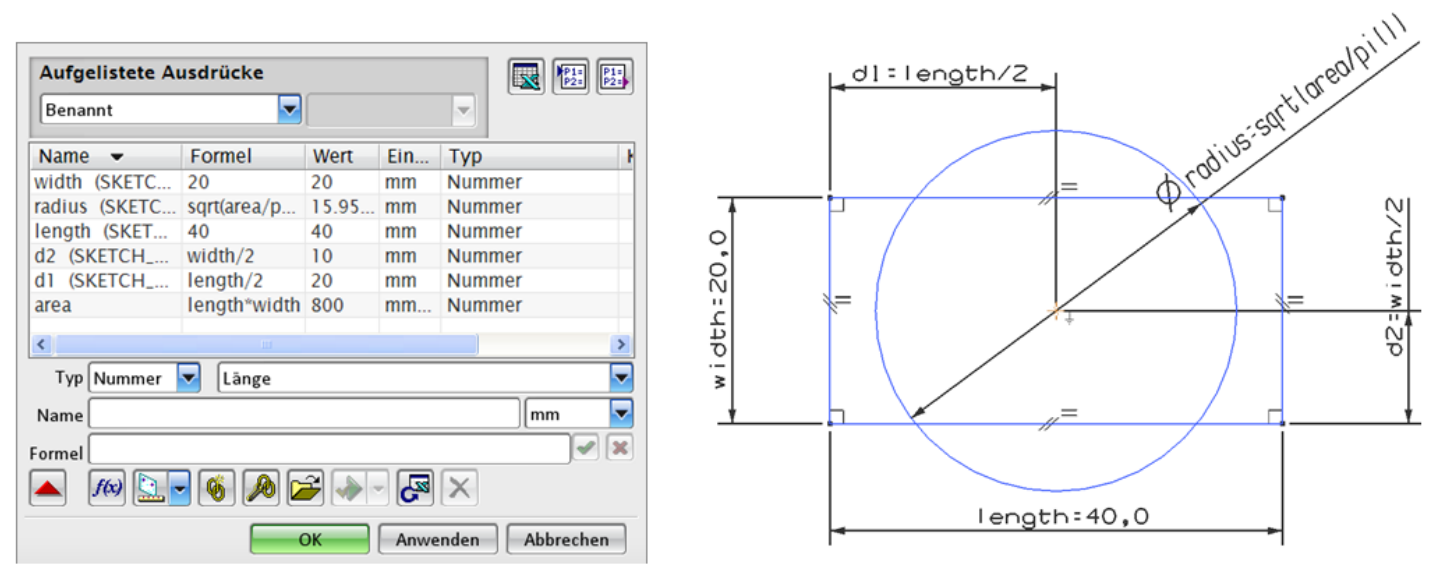

Figure 2. Parameters and constraints of a geometric object

Numerous parametric 3D modeling systems do already exist. Especially in the aircraft and automotive engineering sector, parametric 3D modeling systems are commonly used in daily design processes. In the AEC sector, parametric systems are 
used more and more for designing complex buildings, such as bridges, sports stadiums or hospitals. To determine which system is the most suitable one for modeling complex buildings, a comprehensive CAD study was conducted in the research project 'ForBAU' (Borrmann et al., 2009). In this study, different mechanical and civil engineering systems such as Gehry Technologies Digital Project, Siemens NX and Autodesk Revit Structure have been analyzed and compared. The main results, different diagrams, used modeling methods and further information are summarized in a CAD guide published recently (Obergrießer et al., 2011).

\section{A new approach for integrated geotechnical design and analysis}

The new parametric 3D-model based approach integrates three design/analysis phases and consists of existing as well as newly developed elements (Figure 3). The first phase is concerned with the creation of the roadway (or railway) plan. In the second phase, a parameterized 3D-model of the roadway is created. In the last phase, the information supplied by the 3D-model is used to analyze the geotechnical structure. If changes become necessary, the modified parameters of the roadway are transferred back to the 3D modeling system. The final outcome is an infrastructural and geotechnical building information model (InfraBIM).

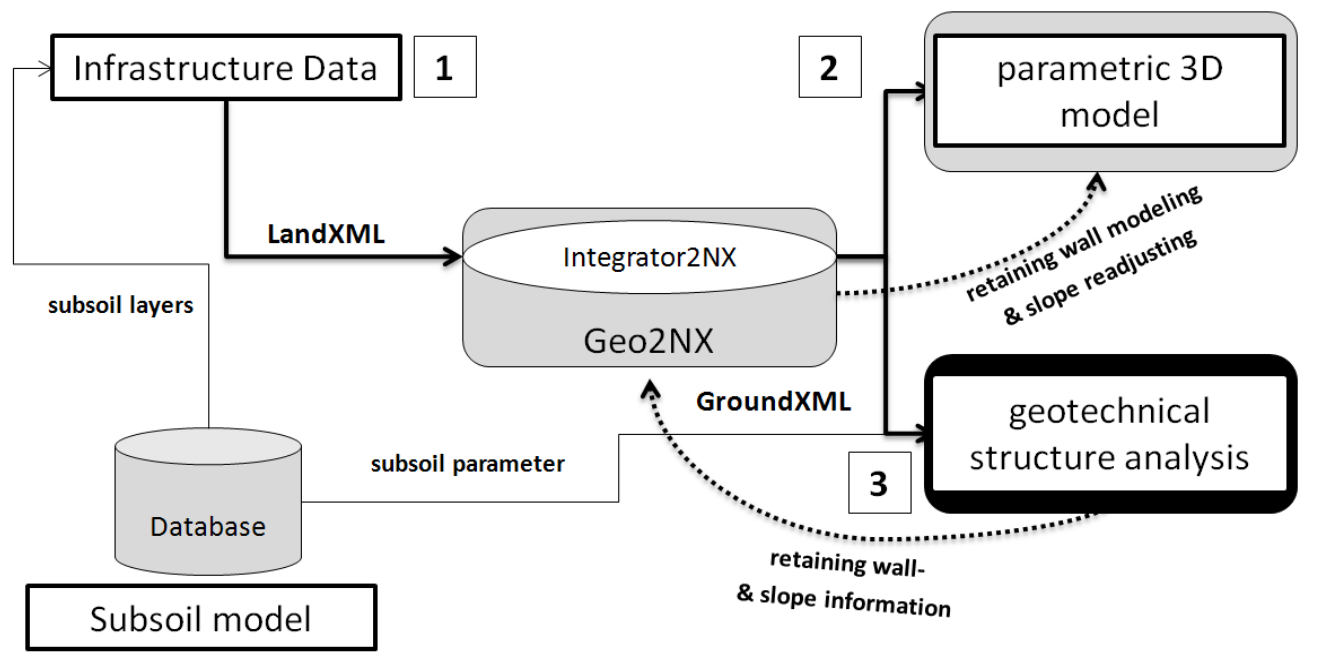

Figure 3. Diagram of the planning steps involved in the proposed integrated process

In Step 1, the initial design of the roadway plan is created using a conventional 2Dbased approach. Hence, the creation of the axis is divided into two 2D steps. At first, the horizontal alignment of the road is designed in the position plan, and in the next step, the vertical alignment is added to the vertical plan. Then different cross sections are generated and added to the alignment. The 2D-based approach is appropriate for designing a roadway because it reduces the complexity of the design by allowing the engineer to concentrate on the main aspects of the different sections. Since the combination of various $2 \mathrm{D}$ sections - horizontal and vertical alignment and the cross section - implicitly describes a 3D model, all required information e.g. 3D points $(x, y$, $\mathrm{z}$ coordinates) of the alignment or different cross sections can be produced as a result of it. The generated cross section plan will now include all of the information on the form of the cutting or dam objects and the position of the surfaces. Unfortunately in 
the $2 \mathrm{D}$ roadway model no additional geotechnical information has been included. After the completion of the roadway design, all the 3D point coordinates and the different cross sections are entered into the 3D modeling system using the LandXML format.

Step 2 of the proposed integration approach involves the creation of a parameterized 3D roadway model. The add-on 'Geo2NX' has been developed for the parametric CAD software Siemens NX to facilitate automatic modeling processes. It generates a volumetric 3D infrastructure model by interpreting the information contained in the LandXML file. The integration processes starts with creating internal objects by parsing the LandXML-File. After this, the 3D alignment curve of the road can be generated as a B-spline object by integrating the 3D points of the alignment as intermediate points of the curve. In the next step, the different cross sections are integrated and automatically parameterized along the curve. At the end of the import phase the generated geometrical objects (B-spline and cross section) are extruded to 3D volumetric objects (Figure 4). Afterwards different Boolean operations are applied to model the final dam and subsoil bodies.

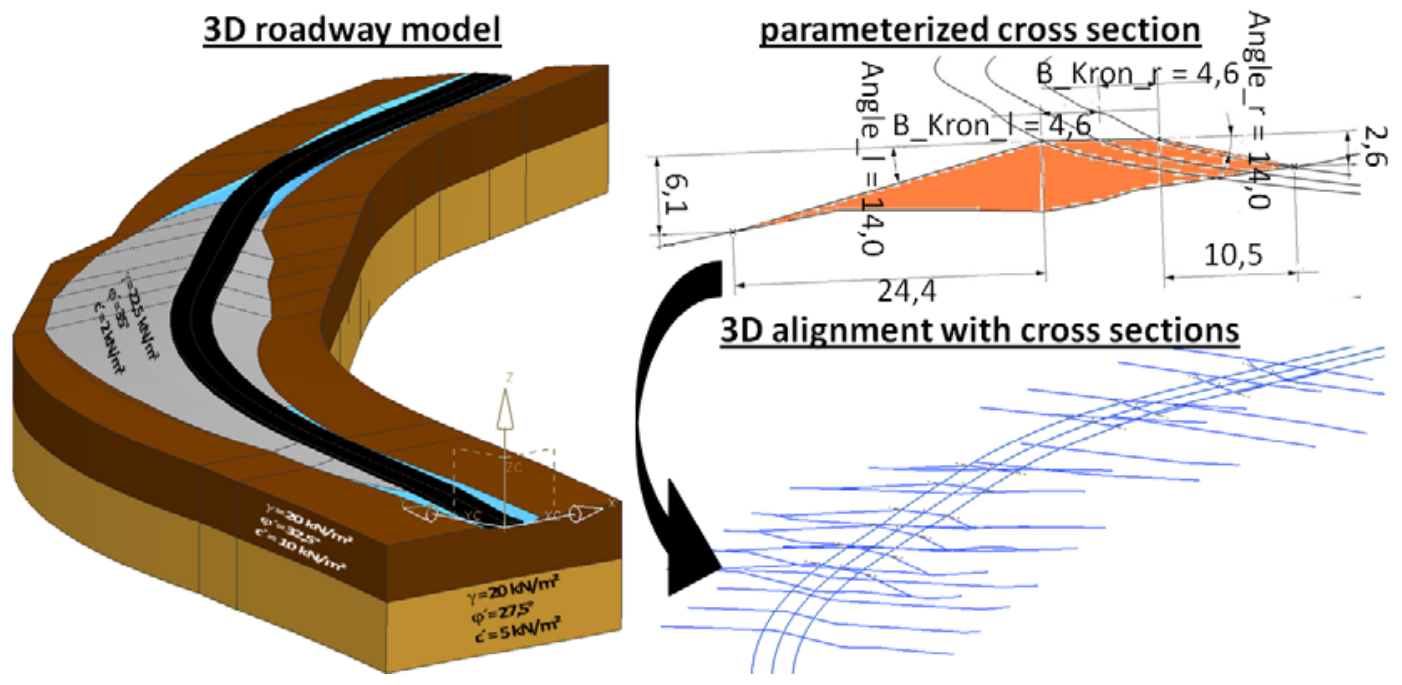

Figure 4. Elements of the parameterized 3D roadway model

Once the modeling phase has been completed the semantic data of the subsoil layers (friction angle, cohesion parameters etc.) is assigned to the different subsoil bodies. The necessary semantic information is provided by the GroundXML format (Obergrießer et al., 2009). Joining the geometric and the semantic information is facilitated by interpreting a subsoil ID that is included in the LandXML and GroundXML format.

The final step of the proposed integrated process is to analyze the geotechnical structure and is illustrated in $2 \mathrm{D}$ by analyzing every significant cross section of the roadway plan. The results of the analyzing process facilitate the engineer to identify the stabile slope angle of the cutting or the dam object and also supply the engineer with information about the position of a required retaining wall or any other kind of geotechnical structure. Currently, the engineer has to enter every cross section manually. This can be avoided by using the geometric and semantic information of the parame- 
terized 3D-model approach. At first, the engineer models the different cross sections into the geomechanical system (Figure 5) by using an interface that automatically interprets and integrates the geometric and semantic information regarding the subsoil and the dam/cut conditions. Then he can start the calculation of each cross section.

The geotechnical analysis may result in the need to change certain parameters of the roadway design. Therefore the modified parameters of the dam or cutting cross sections are transferred back to the 3D modeling system. The exchange of these parameters is facilitated by using an extended version of the LandXML format (Rebolj et al., 2008). Afterwards an update function in the add-on 'Geo2NX' updates the parameter of the 3D roadway model by interpreting the new XML file, for example when the slope angle of a dam is changed from 26.5 degree to 14.0 degree (Figure 6).

In the case that a retaining wall becomes necessary, the 'Geo2NX' module adds the shape of the wall into the existing cross section and automatically generates the body of the retaining wall. At the end of this process a parameterized 3D roadway model that includes all the information of the infrastructural and geotechnical planning process is available.

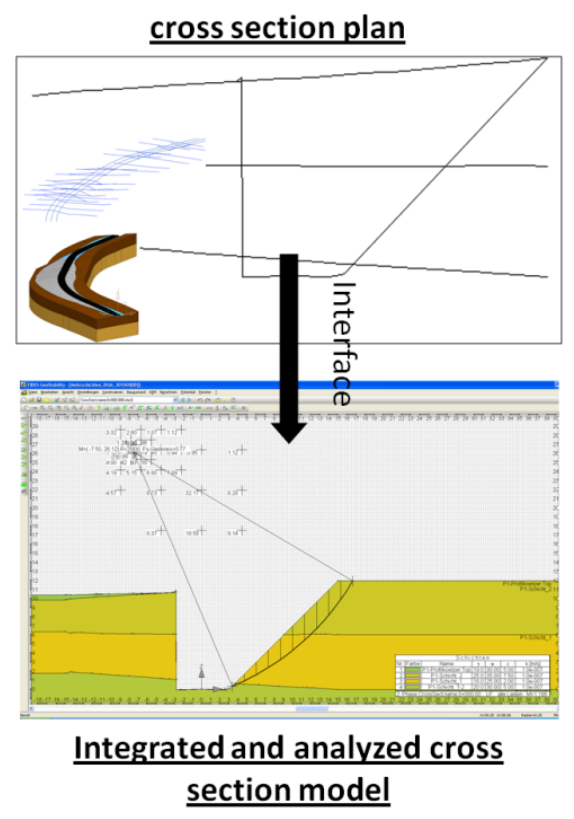

Figure 5. Integrated geotechnical analysis model

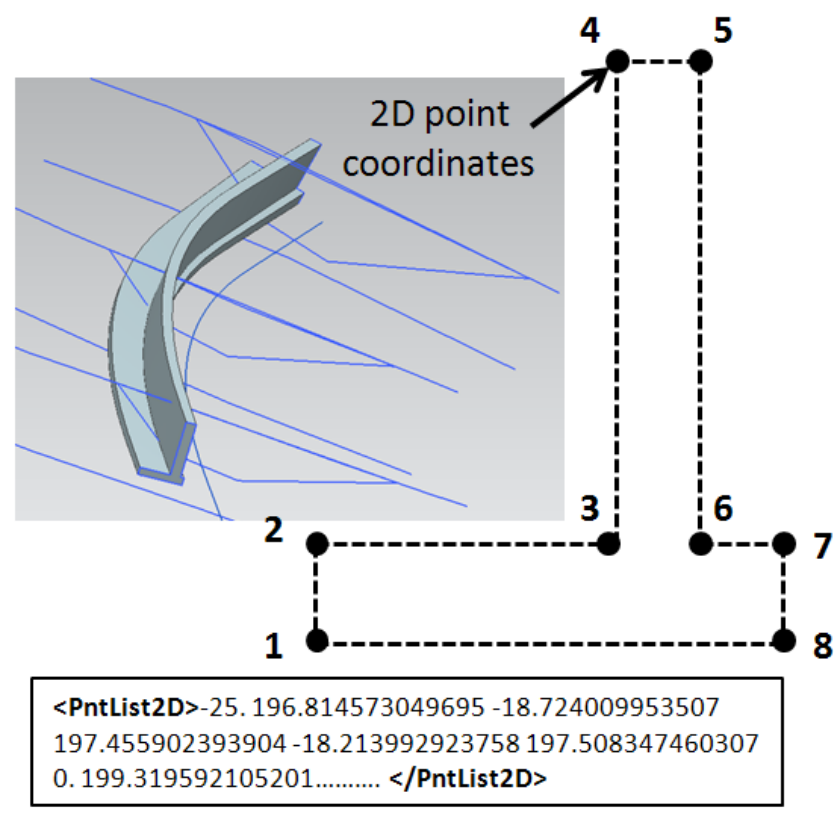

Figure 6. 2D points of the retaining wall

\section{LandXML}

LandXML (www.landxml.org) is a terrestrial and infrastructural extension of the W3C standard XML format and is used to exchange geo-referenced information regarding the surveying and infrastructure planning process. The structure of the data set is defined by the LandXML schema, which is based on the XML format (Crews et al., 2010). Through the hierarchical structure and its easy extensibility, complex datasets can be defined and stored in this format. However, semantic information of 
geotechnical properties such as cohesion parameters or friction angles cannot be transferred using this format.

\section{GroundXML}

GroundXML is an extension of the LandXML format (Obergrießer et al., 2009). It can store all geometrical and semantic information that result from the survey, geotechnical and infrastructure planning processes (Figure 7). The level of the stored data depends on the progress of the planning process. In the first step a GroundXML file is used to transfer the information about the geotechnical data of a 3D subsoil model. In the next step it stores the information about the infrastructure model. Finally it uses the geometrical and semantic infrastructure information to model the cross sections in the geotechnical structural analysis system. The major advantage of this format is that it enables a continuous data stream for the entire planning process.

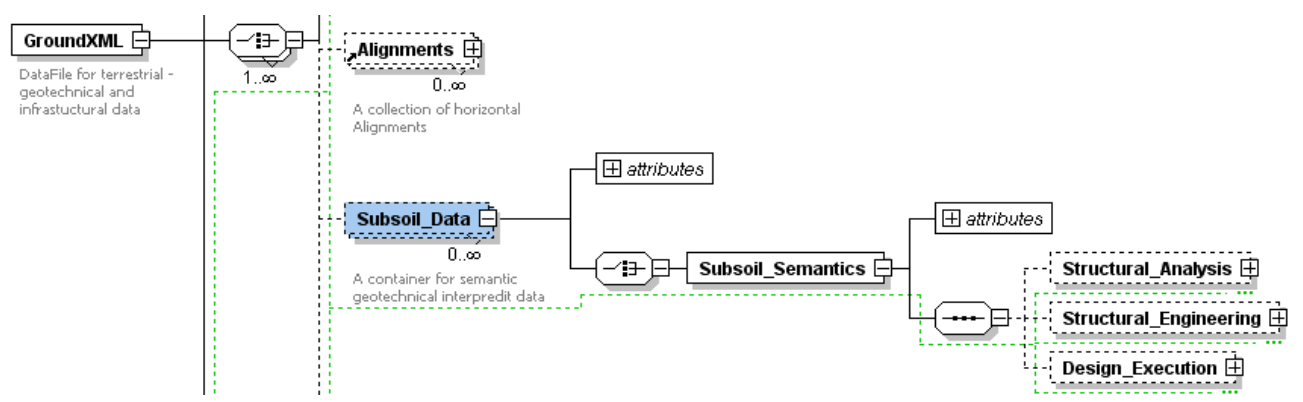

Figure 7. Excerpt of the GroundXML schema

\section{Topological problems}

Some problems have been discovered during the development of the parameterized 3D-model approach. Such problem concerns the topology of the cut and dam cross sections. For this case the cross sections are classified into three different forms
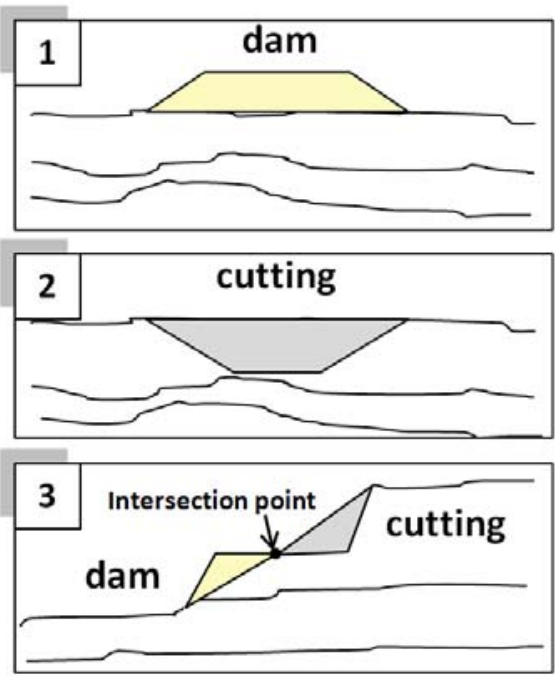

Figure 8. Different cut-dam cross sections
(Figure 8). The first cross section includes only dam geometry and the second one only cut geometry. The third cross section is a mix of a dam and a cut field. It is easy therefore to model a roadway that consists only of a dam or of a cutting cross section because it only requires the extrusion of each cross section along the 3D space curve. An irregular cross section combining dam and cut sections, however, cannot be modeled like this because the left side of the roadway is in a dam area and the right side in a cutting field (or vice versa). The problem is the intersection between the roadway line and the surface line. The existing intersection point results in violating a cross section extrusion rule. To solve this problem an advanced modeling concept has been developed which will be presented in future publications. 


\section{Conclusion}

This 3D-modeling approach helps to improve the infrastructural and geotechnical planning processes. It supports the civil engineer with a parametric $3 \mathrm{D}$ road- or railway product model. Several planning tasks like visualization of the roadway, detecting geometrical collisions, determining earth masses or identifying building managing problems by a 4D simulation can realized by using this unique infrastructural and geotechnical BIM model (InfraBIM). The new approach also closes the information gap between the geometric designing and the geotechnical analyzing process. Furthermore the internal parametrical concept allows updating the InfraBIM model at any time. This leads to a more economical planning process.

As a further work the add-on 'Geo2NX' will be improved by solving the topological problems, creating a possibility to integrate the results of the geotechnical analyses process and refine the LandXML format by integrating geometrical parameters, constraints und further information.

\section{References}

Borrmann, A., Ji, Y., Wu, I-C., Obergrießer, M., Rank, E., Klaubert, C, Günthner, W. (2009). ForBAU - The virtual construction site project. In: Proc. of the 24th CIB-W78 Conference on Managing IT in Construction, Istanbul, Turkey.

Crews, N. and Hall E. (2010). "LandXML Schema." LandXML Schema Version 1.0 Reference. http://www.landxml.org/, (Dec.10, 2010).

Eastman, C., Teicholz, P., Sacks, R., Liston, K. (2008). BIM handbook: A guide to building information modelling for owners, managers, designers, engineers, and contractors, Wiley, New York.

Kaminski, I. (2010). Potenziale des Building Information Modeling im Infrastrukturprojekt - Neue Methoden für einen modellbasierten Arbeitsprozess im Schwerpunkt der Planung, Dissertation, Universtät Leipzig, Leipzig.

Obergrießer, M., Ji, Y., Baumgärtel, T., Euringer, T., Borrmann, A., Rank, E. (2009). GroundXML - An addition of alignment and subsoil specific crosssectional data to the LandXML scheme. In: Proc. of the 12th International Conference on Civil, Structural and Environmental Engineering Computing, Madeira, Portugal.

Obergrießer, M., Euringer, T., Horenburg, T., Günthner, W. (2011). CADModellierung im Bauwesen: Integrierte 3D-Planung von Brückenbauwerken, In: 2. ForBAU Kongress, München. http://www.fml.mw.tum.de/forbau/index.php? Set_ID=496, (Feb.28,2011).

Rebolj, D., Tibaut, A., Čuš-Babič, N., Magdič, A., Podbreznik, P. (2008). „Development and application of a road product model." Automation in construction. Volume 17, Issue 6, 719-72 